\title{
Erratum to: Vicinal Growth as a Possible Structural Source of the Nematic Superconductivity in $\mathrm{Sr}_{x} \mathrm{Bi}_{2} \mathrm{Se}_{3}$
}

\author{
V. P. Martovitskii ${ }^{a, *}$, Yu. G. Selivanov ${ }^{a}$, and A. Yu. Kuntsevich ${ }^{a, b}$ \\ ${ }^{a}$ Lebedev Physical Institute, Russian Academy of Sciences, Moscow, 119991 Russia \\ ${ }^{b}$ Moscow Institute of Physics and Technology, Moscow oblast, Dolgoprudny, 141701 Russia \\ *e-mail: victormart57@mail.ru \\ Received September 13, 2021
}

DOI: $10.3103 / \mathrm{S} 1068335621090037$ lows:

The affiliation of the author Yu. G. Selivanov should be under indication " $a$ " and should read as fol-

${ }^{a}$ Lebedev Physical Institute, Russian Academy of Sciences, Moscow, 119991 Russia

The original article can be found online at

https://doi.org/10.3103/S1068335620040065 